\title{
Review of Particle Physics
}

This article has been downloaded from IOPscience. Please scroll down to see the full text article.

2010 J. Phys. G: Nucl. Part. Phys. 37075021

(http://iopscience.iop.org/0954-3899/37/7A/075021)

View the table of contents for this issue, or go to the journal homepage for more

Download details:

IP Address: 131.215.220.185

The article was downloaded on 21/10/2010 at 16:36

Please note that terms and conditions apply. 


\title{
Review of Particle Physics
}

\author{
K Nakamura et al (Particle Data Group) \\ Online at stacks.iop.org/JPhysG/37/075021
}

\begin{abstract}
This biennial Review summarizes much of particle physics. Using data from previous editions, plus 2158 new measurements from 551 papers, we list, evaluate, and average measured properties of gauge bosons, leptons, quarks, mesons, and baryons. We also summarize searches for hypothetical particles such as Higgs bosons, heavy neutrinos, and supersymmetric particles. All the particle properties and search limits are listed in Summary Tables. We also give numerous tables, figures, formulae, and reviews of topics such as the Standard Model, particle detectors, probability, and statistics. Among the 108 reviews are many that are new or heavily revised including those on neutrino mass, mixing, and oscillations, QCD, top quark, CKM quark-mixing matrix, $V_{u d} \&$ $V_{u s}, V_{c b} \& V_{u b}$, fragmentation functions, particle detectors for accelerator and non-accelerator physics, magnetic monopoles, cosmological parameters, and big bang cosmology.

A booklet is available containing the Summary Tables and abbreviated versions of some of the other sections of this full Review. All tables, listings, and reviews (and errata) are also available on the Particle Data Group website: pdg.lbl.gov.
\end{abstract}

The 2010 edition of Review of Particle Physics is published for the Particle Data Group as article 075021 in volume 37 of Journal of Physics G: Nuclear and Particle Physics.

This edition should be cited as:

K Nakamura et al (Particle Data Group) 2010 J. Phys. G: Nucl. Part. Phys. 37075021

\section{ACCESS TO FULL TEXT PDF}

$\begin{array}{ll}\text { PDF (168 KB) } & \text { Abstract, Contributors, Highlights and Table of Contents } \\ \text { PDF (482 KB) } & \text { Introduction } \\ & \text { Particle Physics Summary Tables } \\ \text { PDF (189 KB) } & \text { Gauge and Higgs Bosons } \\ \text { PDF (171 KB) } & \text { Leptons } \\ \text { PDF (102 KB) } & \text { Quarks } \\ \text { PDF (893 KB) } & \text { Mesons } \\ \text { PDF (379 KB) } & \text { Baryons } \\ \text { PDF (112 KB) } & \text { Searches (Supersymmetry, Compositeness, etc) } \\ \text { PDF (381 KB) } & \text { Tests of Conservation Laws }\end{array}$


PDF (55 KB)

PDF (396 KB)

PDF (5.58 MB)

PDF (1.71 MB)

PDF (4.04 MB)

PDF $(711 \mathrm{~KB})$

PDF (957 KB)

PDF (297 KB)

PDF (2.31 MB)

PDF (1.80 MB)

PDF (948 KB)

PDF (4.44 MB)

PDF (7.02 MB)

PDF (9.46 MB)

PDF (5.72 MB)

PDF (5.41 MB)

PDF (194 KB)

PDF (4.24 MB)

\section{Reviews, Tables and Plots}

Detailed contents for this section

Constants, Units, Atomic and Nuclear Properties

Standard Model and Related Topics

Astrophysics and Cosmology

Experimental Methods and Colliders

Mathematical Tools or Statistics, Monte Carlo, Group Theory

Kinematics, Cross-Section Formulae, and Plots

\section{Particle Listings}

Introduction: Illustrative Key and Abbreviations

Gauge and Higgs Bosons

Leptons

Quarks

Mesons: Light unflavored and strange

Mesons: Charmed and bottom

Mesons: Other

Baryons

Miscellaneous Searches

\section{Index}

Color Figures 